\title{
Violência obstétrica e prevenção quaternária: o que é e o que fazer
}

\author{
Obstetric violence and quaternary prevention: what it is and what to do
}

\section{La violencia obstétrica y la prevención cuaternaria: qué es y qué hacer}

Charles Dalcanale Tesser. Departamento de Saúde Pública. Universidade Federal de Santa Catarina (UFSC). Florianópolis, SC, Brasil. charles.tesser@ufsc.br (Autor correspondente)

Roxana Knobel. Departamento de Tocoginecologia. Universidade Federal de Santa Catarina (UFSC). Florianópolis, SC, Brasil. rknobel@uol.com.br Halana Faria de Aguiar Andrezzo. Faculdade de Saúde Pública. Universidade de São Paulo (USP). São Paulo, SP, Brasil. halanaf@hotmail.com Simone Grilo Diniz. Departamento de Saúde Materno-Infantil. Faculdade de Saúde Pública. Universidade de São Paulo (USP). São Paulo, SP, Brasil. sidinizg@gmail.com

\section{Resumo}

0 objetivo deste artigo é justificar a necessidade de prevenção quaternária frente à 'violência obstétrica' (VO), expressão que agrupa todas as formas de violência e danos originados no cuidado obstétrico profissional, bem como discutir estratégias e ações de prevenção quaternária a serem realizadas pelos médicos de família e comunidade (MFC), pelas equipes de atenção primária à saúde (APS) e suas entidades associativas. A prevalência de violência obstétrica no Brasil é alta: 1/4 das mulheres relata terem sofrido maus-tratos durante 0 atendimento ao parto, além de excesso de intervenções desnecessárias (como venóclise, ocitocina de rotina e episiotomia) e privação de uma assistência baseada em boas práticas, tais como parto em posição verticalizada, possibilidade de se alimentar e de se movimentar durante 0 trabalho de parto e presença de um acompanhante. Destaca-se 0 excesso crônico de cesarianas (55,6\% do total de nascimentos) no Brasil, mais prevalente no setor privado (85\%) do que no público (40\%). Ações de prevenção quaternária dirigidas à V0 são propostas e discutidas, como: (1) a elaboração (individual e coletiva) de planos de parto orientados pelas equipes de APS no pré-natal (para os quais se oferece um roteiro); (2) a introdução de outros profissionais qualificados no cuidado ao parto de risco habitual (incluindo MFC capacitados); e (3) a participação dos MFC e profissionais da APS e suas associações no movimento social e político pela "humanização" do parto, com apoio às mudanças nas maternidades já em funcionamento e às novas iniciativas de serviços de cuidado ao parto.

\section{Abstract}

This article aims to justify the necessity of quaternary prevention in face of 'obstetric violence' (OV), expression that comprises all forms of harms and violence originated by professional obstetric care, as well as to discuss actions and strategies of quaternary prevention to be taken by family physicians primary care providers and their professional associations. The prevalence of obstetric violence in Brazil is high: $1 / 4$ of women report that they have suffered abusive treatment during birth delivery, besides the excess of unnecessary interventions (i.e. venoclisis, routine oxitocin, and episiotomy), consequently denying them a best practice care such as vertical position, allowing the pregnant woman to freely move, eat, and have a companion during labour process. There is an excess of caesareans (55.6\% of the total births) in Brazil, most prevalent on the private sector (85\%) than in the public health system (40\%). We propose and discuss actions of quaternary prevention against obstetric violence: (1) the elaboration (individual and collective) of birth plans oriented by primary care teams during antenatal care (for which we suggest a guideline); (2) the introduction of other qualified professionals on the caring for low risk birth (including qualified family physicians); and (3) the participation of family physicians and other primary care providers and their associations on the social and political movement for "humanization of birth", supporting the changes on currently functioning maternity wards and new initiatives on birth delivery care.
Palavras-chave:

Violência contra a Mulher Doença latrogênica Prevenção Quaternária Autonomia Pessoal Parto Obstétrico

\section{Keywords:} Violence Against Women latrogenic Disease Quaternary Prevention Personal Autonomy Delivery, Obstetric 


\section{Resumen}

El objetivo de este artículo es justificar la necesidad de la prevención cuaternaria frente a la 'violencia obstétrica' (V0), expresión que agrupa a todas las formas de violencia y los daños derivados de la atención obstétrica profesional, así como para discutir las estrategias y acciones de prevención cuaternaria que podrían ser adoptadas por los médicos de Familia y comunidad (MFC), por los equipos de atención primaria de salud (APS) y sus entidades asociativas. La prevalencia de violencia obstétrica en Brasil es alta: $1 / 4$ de las mujeres informó haber sufrido malos tratos durante el trabajo de parto, así como excesivas intervenciones innecesarias (como las venoclisis, la oxitocina de rutina y la episiotomía) y la privación de una asistencia basada en las mejores prácticas, tales como el parto en una posición vertical, la posibilidad de alimentarse y desplazarse durante el parto y la presencia de un acompañante. Destacamos el exceso crónico de cesáreas (55,6\% de todos los nacimientos) en Brasil, más frecuente en el sector privado (85\%) que en el sector público (40\%). Acciones de prevención cuaternaria dirigidas a la violencia obstétrica son propuestas y discutidas, tales como: (1) la preparación (individual y colectiva) de los planes de parto guiados por los equipos de APS en la atención prenatal (que prevé un plan de trabajo); (2) la introducción de otros profesionales calificados en el cuidado del parto de bajo riesgo (incluidos MFC entrenados); y (3) la participación de médicos familiares y profesionales de la APS y sus asociaciones en el movimiento social y político para la "humanización" del parto, con soporte para los cambios en las maternidades en funcionamiento y las nuevas iniciativas de los servicios de atención al parto.

\section{Palabras clave:}

Violencia contra la Mujer Enfermedad latrogénica Prevención Cuaternaria Autonomía Personal

Parto Obstétrico

\section{Introdução}

Adelir, 29 anos, G3POC2A0, casada, em abril de 2014 estava no final da gestação e desejava parto normal. Fez o pré-natal no centro de saúde, mas abandonou o seguimento com 39 semanas por medo de ser induzida a uma cesariana. Com 41 semanas de gestação, foi ao hospital para uma avaliação de vitalidade fetal e iniciando o trabalho de parto. Foi realizado um ultrassom que mostrou perfeitas condiçóes de vitalidade fetal e feto pélvico. A médica no hospital indicou cesariana, por duas cirurgias anteriores e feto pélvico. Adelir recusou, assinou termo de responsabilidade e voltou para casa, aguardar o avanço de seu trabalho de parto. Foi surpreendida às 01:30h por policiais que a obrigaram a ir ao hospital realizar a cesariana, enviados por um juiz, acionado por um promotor, requisitado pelo hospital onde tinha sido atendida. No hospital, o marido é impedido de acompanhar a cesariana. Não houve intercorrências e o bebê nasceu com boa vitalidade..$^{1,2}$

A expressão "violência obstétrica" (VO) é utilizada para descrever e agrupar diversas formas de violência (e danos) durante o cuidado obstétrico profissional. Inclui maus tratos físicos, psicológicos, e verbais, assim como procedimentos desnecessários e danosos - episiotomias, restrição ao leito no pré-parto, clister, tricotomia e ocitocina (quase) de rotina, ausência de acompanhante - dentre os quais destaca-se o excesso de cesarianas, crescente no Brasil há décadas, apesar de algumas iniciativas governamentais a respeito. ${ }^{3}$ A história de Adelir, por exemplo, sintetizada acima, apesar de circular na mídia em vários países, não gerou manifestaçôes sociais ou de entidades médicas brasileiras. Apenas ativistas da luta pela 'humanização' do parto criticaram a violência, e não se tem informação de denúncias realizadas junto à justiça comum ou ao Conselho Regional de Medicina.

Recente pesquisa nacional deu visibilidade ao problema: cerca de $1 / 4$ das mulheres que tinham parido, e também aproximadamente metade das que abortaram, relataram alguma forma de VO. ${ }^{4}$ Esses fatos são a ponta de um iceberg com o qual a sociedade e os profissionais da atenção primária à saúde (APS) brasileira têm convivido passivamente. Sendo a prevenção quaternária a atitude e a ação de identificação e evitação de risco de hipermedicalização, intervençôes desnecessárias e danos, ${ }^{5}$ ela deve considerar a VO em seu escopo de saberes, valores e práticas.

O objetivo deste artigo é justificar a necessidade de prevenção quaternária frente à 'violência obstétrica' (VO), expressão que agrupa todas as formas de violência e danos originados no cuidado obstétrico profissional, bem como discutir estratégias e açôes de prevenção quaternária a serem realizadas pelos médicos de família e comunidade (MFC), pelas equipes de atenção primária à saúde (APS) e suas entidades associativas. A VO diz respeito a vários profissionais. No Brasil, a quase totalidade dos partos são hospitalares (em 2012, 98\% dos 2.905 .789 nascimentos com nascidos vivos ocorreram em hospital ${ }^{6}$ ), atendidos em sua maioria por médicos obstetras. Porém, este artigo é voltado aos profissionais da atenção primária à saúde (especialmente aos MFC), responsáveis pelo pré-natal de grande parte das gestantes do país. O texto está estruturado em três partes: a primeira trata da $\mathrm{VO}$; a segunda aborda intervençóes prejudiciais na parturição e o excesso de cesarianas no Brasil; e a terceira finaliza com açōes que os MFC e demais profissionais da APS poderiam realizar como estratégia para a prevenção quaternária. 


\section{Violência obstétrica}

$\mathrm{Na}$ última década, várias definiçôes de violência obstétrica têm sido propostas. Uma delas é a da primeira legislação latino-americana tipificando esta forma de violência, aprovada na Venezuela:

Qualquer conduta, ato ou omissão por profissional de saúde, tanto em público como privado, que direta ou indiretamente leva à apropriaçấo indevida dos processos corporais e reprodutivos das mulheres, e se expressa em tratamento desumano, no abuso da medicalização e na patologização dos processos naturais, levando à perda da autonomia e da capacidade de decidir livremente sobre seu corpo e sexualidade, impactando negativamente a qualidade de vida de mulheres (p.30). ${ }^{7}$

Várias expressóes já foram usadas para designar o fenômeno, como "violência no parto", "abuso obstétrico", "desrespeito e abuso", 8 "violência de gênero no parto e aborto", "violência institucional de gênero no parto e aborto", " "assistência desumana/desumanizada", "crueldade no parto", ${ }^{10}$ "violaçóes dos Direitos Humanos das mulheres no parto". ${ }^{11}$ Dentre as várias conceituaçóes possíveis, a proposta por Bowser e Hill ${ }^{8}$ elenca as principais categorias de desrespeito e abuso nas instituições de saúde, associando-as aos direitos correspondentes, sintetizadas no Quadro 1.

O Quadro 1 demonstra a existência de uma superposição entre VO e dano iatrogênico no parto. Uma forma pragmática de visibilizar esses danos é o "termômetro de segurança da assistência materna", recentemente criado pelo National Health Service (NHS) inglês, ${ }^{12}$ que registra danos medidos individualmente em uma dada população (Quadro 2).

Estes indicadores podem ser usados pela vigilância sanitária, para medir o resultado da assistência em um determinado serviço ou região de saúde. No contexto da saúde materna e perinatal, a prática da prevenção quaternária é indissociável do cuidado baseado em evidências científicas, da humanização do parto e do combate à VO, que deve ser encarada como questấo prioritária, "pois representa a desumanização do cuidar e a perpetuação do ciclo de opressão feminina pelo próprio sistema de saúde” (p. S12). ${ }^{13}$

Quadro 1. Categorias de violência obstétrica, direitos e exemplos.

\begin{tabular}{|c|c|c|}
\hline Categoria & Direito correspondente & Situações exemplares \\
\hline Abuso físico. & $\begin{array}{l}\text { Direito a estar livre de tratamento } \\
\text { prejudicial e de maus tratos. }\end{array}$ & $\begin{array}{l}\text { Procedimentos sem justificativa clínica e intervenções "didáticas", como toques vaginais } \\
\text { dolorosos e repetitivos, cesáreas e episiotomias desnecessárias. Imobilização física em posições } \\
\text { dolorosas, prática da episiotomia e outras intervenções sem anestesia, sob a crença de que a } \\
\text { paciente "já está sentindo dor mesmo". }\end{array}$ \\
\hline $\begin{array}{l}\text { Imposição de intervenções } \\
\text { não consentidas. Intervenções } \\
\text { aceitas com base em } \\
\text { informações parciais ou } \\
\text { distorcidas. }\end{array}$ & $\begin{array}{l}\text { Direito à informação, ao } \\
\text { consentimento informado e à } \\
\text { recusa, e respeito pelas escolhas } \\
\text { e preferências, incluindo } \\
\text { acompanhantes durante } 0 \\
\text { atendimento de maternidade. }\end{array}$ & $\begin{array}{l}\text { Mulheres que verbalmente e por escrito, não autorizam uma episiotomia, mas esta intervenção } \\
\text { é feita à revelia da sua desautorização. Recusa à aceitação de planos de parto. Indução à } \\
\text { cesárea por motivos duvidosos, tais como superestimação dos riscos para o bebê (circular } \\
\text { de cordão, "pós-datismo" na } 40^{a} \text { semana, etc.) ou para a mãe (cesárea para "prevenir danos } \\
\text { sexuais", etc.). Não informação dos danos potenciais de longo prazo dos modos de nascer } \\
\text { (aumento de doenças crônicas nos nascidos, por exemplo). }\end{array}$ \\
\hline $\begin{array}{l}\text { Cuidado não confidencial ou } \\
\text { privativo. }\end{array}$ & Confidencialidade e privacidade. & $\begin{array}{l}\text { Maternidades mantêm enfermarias de trabalho de parto coletivas, muitas vezes sem sequer } \\
\text { um biombo separando os leitos, e ainda usam a falta de privacidade como justificativa para } \\
\text { desrespeitar o direito a acompanhantes. }\end{array}$ \\
\hline Cuidado indigno e abuso verbal. & Dignidade e respeito. & $\begin{array}{l}\text { Formas de comunicação desrespeitosas com as mulheres, subestimando e ridicularizando sua } \\
\text { dor, desmoralizando seus pedidos de ajuda. Humilhações de caráter sexual, do tipo "quando } \\
\text { você fez você achou bom, agora está aí chorando". }\end{array}$ \\
\hline $\begin{array}{l}\text { Discriminação baseada em } \\
\text { certos atributos. }\end{array}$ & $\begin{array}{l}\text { Igualdade, não discriminação, } \\
\text { equidade da atenção. }\end{array}$ & $\begin{array}{l}\text { Tratamento diferencial com base em atributos considerados positivos (casadas, com gravidez } \\
\text { planejadas, adultas, brancas, mais escolarizadas, de classe média, saudáveis, etc.) depreciando } \\
\text { as que têm atributos considerados negativos (pobres, não-escolarizadas, mais jovens, negras, e } \\
\text { as que questionam ordens médicas). }\end{array}$ \\
\hline $\begin{array}{l}\text { Abandono, negligência ou } \\
\text { recusa de assistência. }\end{array}$ & $\begin{array}{l}\text { Direito ao cuidado à saúde em } \\
\text { tempo oportuno e ao mais alto nível } \\
\text { possível de saúde. }\end{array}$ & $\begin{array}{l}\text { Estudos mostram o abandono, a negligência ou recusa de assistência às mulheres que } \\
\text { são percebidas como muito queixosas, descompensadas ou demandantes, e nos casos } \\
\text { de assistência ao aborto incompleto, frequentemente são deixadas por último, com riscos } \\
\text { importantes à sua segurança física. }\end{array}$ \\
\hline Detenção nos serviços. & Liberdade, autonomia. & $\begin{array}{l}\text { Pacientes podem ficar retidas até que saldem as dívidas com os serviços. No Brasil e em outros } \\
\text { países, começam a ocorrer detenções policiais, como no caso narrado no início deste artigo. }\end{array}$ \\
\hline
\end{tabular}

Fonte: elaborado a partir de Bowser e Hill. ${ }^{8}$ 
Quadro 2. Termômetro de segurança da assistência materna do NHS.

\begin{tabular}{|l|}
\hline Dano perineal (laceração e episiotomia) \\
\hline Trauma abdominal (cesariana ou laparotomia) \\
\hline Hemorragia pós-parto \\
\hline Infecção (trato urinário, mastite, ferida perineal, de cesária, laparotomia ou uterina) \\
\hline Desfechos do bebê: apgar <7 (5 min.) \\
\hline Transferência do serviço ou internação em UTI neonatal \\
\hline $\begin{array}{l}\text { Segurança emocional da mãe (separação mãe-bebê, ser deixada sozinha pela equipe quando preocupada, preocupação sobre segurança durante assistência levada em } \\
\text { consideração, dúvida que não foi sanada) }\end{array}$ \\
\hline
\end{tabular}

Fonte: Safety Thermometer, National Health Service. ${ }^{12}$

\section{Excesso de intervenções no parto e cesáreas no Brasil}

Em 1996, a publicação da OMS "Assistência ao parto normal: um guia prático"14 classificou as intervençôes durante o parto conforme evidências científicas, o que foi amplamente divulgado no Brasil, em 2001. ${ }^{15}$ As práticas claramente prejudiciais ou ineficazes e que devem ser eliminadas estão sumarizadas no Quadro 3.

Quadro 3. Práticas prejudiciais e motivos associados.

\begin{tabular}{|c|c|}
\hline Práticas prejudiciais ou ineficazes & Motivo \\
\hline $\begin{array}{l}\text { Infusão intravenosa de rotina no trabalho de parto/ } \\
\text { Cateterização venosa profilática de rotina. }{ }^{13}\end{array}$ & $\begin{array}{l}\text { Diminui a mobilidade, "prende" a parturiente ao leito. }{ }^{16} \\
\text { Aumenta desconforto. }{ }^{16} \\
\text { Solução glicosada pode aumentar a possibilidade de hipoglicemia neonatal. }{ }^{16}\end{array}$ \\
\hline Uso indiscriminado de ocitocina. & $\begin{array}{l}\text { Pode levar a um aumento da atividade uterina com conseqüente hipóxia fetal. }{ }^{15} \\
\text { Ocitocina isoladamente não diminui a possibilidade de cesariana em mulheres com analgesia peridural. }{ }^{17}\end{array}$ \\
\hline Amniotomia para acelerar trabalho de parto. & $\begin{array}{l}\text { Amniotomia isolada parece diminuir um pouco a duração do trabalho de parto, mas aumenta a possibilidade de } \\
\text { cesariana. }^{18}\end{array}$ \\
\hline $\begin{array}{l}\text { Posição de litotomia (posição de exame } \\
\text { ginecológico). }\end{array}$ & $\begin{array}{l}\text { Posições verticalizadas reduzem o tempo de trabalho de parto e não estão associadas a aumento de intervenções } \\
\text { ou efeitos negativos }{ }^{19} \\
\text { São bem descritos os benefícios da posição verticalizada para mulher e feto. }{ }^{19,20}\end{array}$ \\
\hline Episiotomia. & $\begin{array}{l}\text { Aumenta o risco de laceração perineal de terceiro e quarto graus, de infecção e de hemorragia, sem diminuir } \\
\text { complicações a longo prazo de dor e incontinência urinária e fecal. }{ }^{21} \\
\text { Seu uso rotineiro vir sendo constantemente desestimulado. }{ }^{22}\end{array}$ \\
\hline Manobra de Kristeller. & $\begin{array}{l}\text { Associada a lacerações perineais graves e internação em UTI neonatal para o bebê. }{ }^{23} \\
\text { Há recomendação de que seja evitada. }{ }^{24}\end{array}$ \\
\hline Restrição alimentar e hídrica. & $\begin{array}{l}\text { Prolongada pode levar a desconforto da parturiente, há recomendação de que as mulheres tenham liberdade para } \\
\text { ingerir líquidos e outros alimentos leves durante } 0 \text { trabalho de parto. }{ }^{25}\end{array}$ \\
\hline Restrição aos movimentos corporais. & $\begin{array}{l}\text { Dificulta lidar com a dor. }{ }^{26} \\
\text { Aumenta a chance de necessidade de analgesia. }{ }^{26} \\
\text { Aumenta a chance de cesariana. }{ }^{26} \\
\text { Aumenta a duração do trabalho de parto. }{ }^{26}\end{array}$ \\
\hline Impedimento de acompanhante. & $\begin{array}{l}\text { Presença de acompanhantes é altamente protetora contra todas as formas de violência durante a internação } \\
\text { hospitalar. }{ }^{27}\end{array}$ \\
\hline
\end{tabular}

Fonte: elaborado pelos autores a partir das fontes referenciadas na tabela.

A recente pesquisa Nascer no Brasil, ${ }^{28}$ contemplando uma amostra representativa dos partos hospitalares de todo o país (266 maternidades públicas e privadas com 500 ou mais partos anuais em 191 municípios) entrevistou mais de 23 mil mulheres e mostrou que as práticas prejudiciais/ineficazes acima mencionadas ainda são rotina no país. Entre as entrevistadas, $70 \%$ foram rotineiramente puncionadas, $40 \%$ receberam ocitocina e realizou-se amniotomia em $40 \%$. Entre as mulheres que pariram (48\% da amostra), 92\% estavam em posição de litotomia (deitadas), 56\% foram submetidas a epsiotomia, 
$37 \%$ receberam a manobra de Kristeller (aplicação de pressão na parte superior do útero durante o período expulsivo). Somente $26 \%$ puderam se alimentar, $46 \%$ puderam se movimentar durante o trabalho de parto e $18,7 \%$ contaram com acompanhante. Apenas 5\% tiveram partos sem nenhuma intervenção. Não são necessários comentários adicionais para enfatizar a magnitude da $\mathrm{VO}$ no país.

\section{Cesariana: quando esta se torna uma violência obstétrica}

A cirurgia cesariana, quando necessária, salva vidas e diminui morbidades para a mulher e o concepto. ${ }^{29}$ Todavia, esse efeito positivo não se mantém a partir de certa proporção de cesarianas, mesmo em populaçôes com acesso a todos os cuidados necessários intra e pós-cirúrgicos. Em 1985, a Organização Mundial da Saúde (OMS) definiu que o índice máximo de cesarianas deve ser de $15 \%{ }^{30}$ Embora não haja consenso sobre um número aceitável ou ideal, é injustificável clinicamente uma taxa global superior a $10-15 \% .{ }^{31}$

A melhoria de técnicas cirúrgicas, anestésicas, de antibioticoterapia e de cuidados intensivos criaria uma expectativa de que eventuais efeitos adversos e complicaçôes estivessem diminuindo. No entanto, os efeitos adversos da cesariana são evidentes e persistentes e estudos demonstram que o aumento nas taxas de cesariana isoladamente não obteve efeito na redução da mortalidade perinatal. ${ }^{32} \mathrm{O}$ decréscimo na mortalidade perinatal se deve, possivelmente, a avanços no cuidado pré-natal, uso de corticosteróides para maturidade pulmonar, melhorias no cuidado intensivo ao recém-nascido, não podendo ser explicado apenas pelo aumento de cesarianas. ${ }^{32}$

Nas cesariadas, em relação às mulheres que tiveram partos normais, há aumento de mortalidade, morbidade severa, internação em UTI, uso de antibióticos, necessidade de transfusão, histerectomia e tempo de permanência no hospital. ${ }^{33}$ A possibilidade de complicaçôes se torna mais evidente em gestações futuras, com um aumento do risco de placentação anômala e suas graves conseqüências. ${ }^{34}$ Em bebês que nascem de cesariana há maior risco de prematuridade, mortalidade neonatal, admissão em UTI neonatal e uso de ventilação mecânica. ${ }^{32,33,35,36}$ A prematuridade iatrogênica e o nascimento eletivo de bebês antes de 39 semanas causa aumento de internaçóes em UTI neonatal e do número de óbitos. ${ }^{36,37}$ No Brasil, estudos mostraram a relação entre o aumento de conceptos prematuros e de baixo peso com as taxas aumentadas de cesariana. ${ }^{38,39}$

As evidências da importância fundamental da gravidez, parto e nascimento na promoção da saúde na vida adulta vem se avolumando. Pesquisas epidemiológicas mostram que, em comparação com os nascidos de parto vaginal, os nascidos de cesárea têm um risco aumentado de sobrepeso e obesidade, diabetes tipo 1, asma, alergias digestivas e de pele, entre outros problemas relacionados às características da imunidade e do metabolismo. ${ }^{40}$ Uma revisão sistemática destes estudos confirma estas tendências, mesmo controlando os potenciais fatores de confusão. ${ }^{41}$

O trabalho de parto e o parto são potentes processos neuroendócrinos que interferem na expressão do genoma humano (processos epigenéticos). Os mecanismos de regulação do stress, a produção de neurotransmissores e a transição respiratória são distintas para os que passaram e não passaram pelo trabalho de parto, com consequências epigenéticas que podem durar toda a vida. ${ }^{42,43} \mathrm{O}$ modo de nascer tem repercussóes para toda a vida sobre o risco de doenças crônicas, e há evidências sólidas destas diferenças.

Em geral, há grande excesso de realização de cesarianas no Brasil, sendo mais prevalente no setor suplementar (assim como em países e populaçóes mais ricas no mundo). Em 2009, no Brasil, o número de nascidos vivos por cirurgia excedeu os nascidos vivos por parto normal. ${ }^{39}$ Estima-se que em 2012 as taxas foram de 55,6\% no país, 40\% nos serviços públicos e $85 \%$ nos serviços privados. ${ }^{6}$ No Gráfico 1 são apresentadas as proporçóes entre partos normais e cesarianas entre serviços púbicos e privados, evidenciando uma tendência de piora nos últimos anos.

Para se compreender o fenômeno do abuso de cesarianas deve-se considerar a multiplicidade e a complexidade dos fatores envolvidos. Há fatores culturais que se referem à medicalização do ideário das pessoas e das mulheres, fazendo com que a cesariana seja considerada um bem de consumo e uma intervenção segura. ${ }^{44,45}$ Embora algumas mulheres realmente escolham essa via de parto, esta não é a escolha da maioria das usuárias. ${ }^{46,47}$ As que preferem a cesariana são influenciadas por fatores culturais (crença histórica e errônea de que tem impacto na diminuição da mortalidade perinatal, medo do parto normal ${ }^{48}$ e experiências negativas com partos anteriores ${ }^{49}$ ) e pelos médicos que as assistem. ${ }^{44,50}$ Entrevistas realizadas com mulheres de classe média que foram cesariadas evidenciaram que os médicos determinam a via de parto quer seguindo o desejo das mulheres (quando elas desejam a cirurgia) ou montando um cenário de necessidade clínica da cesariana. ${ }^{51}$ 


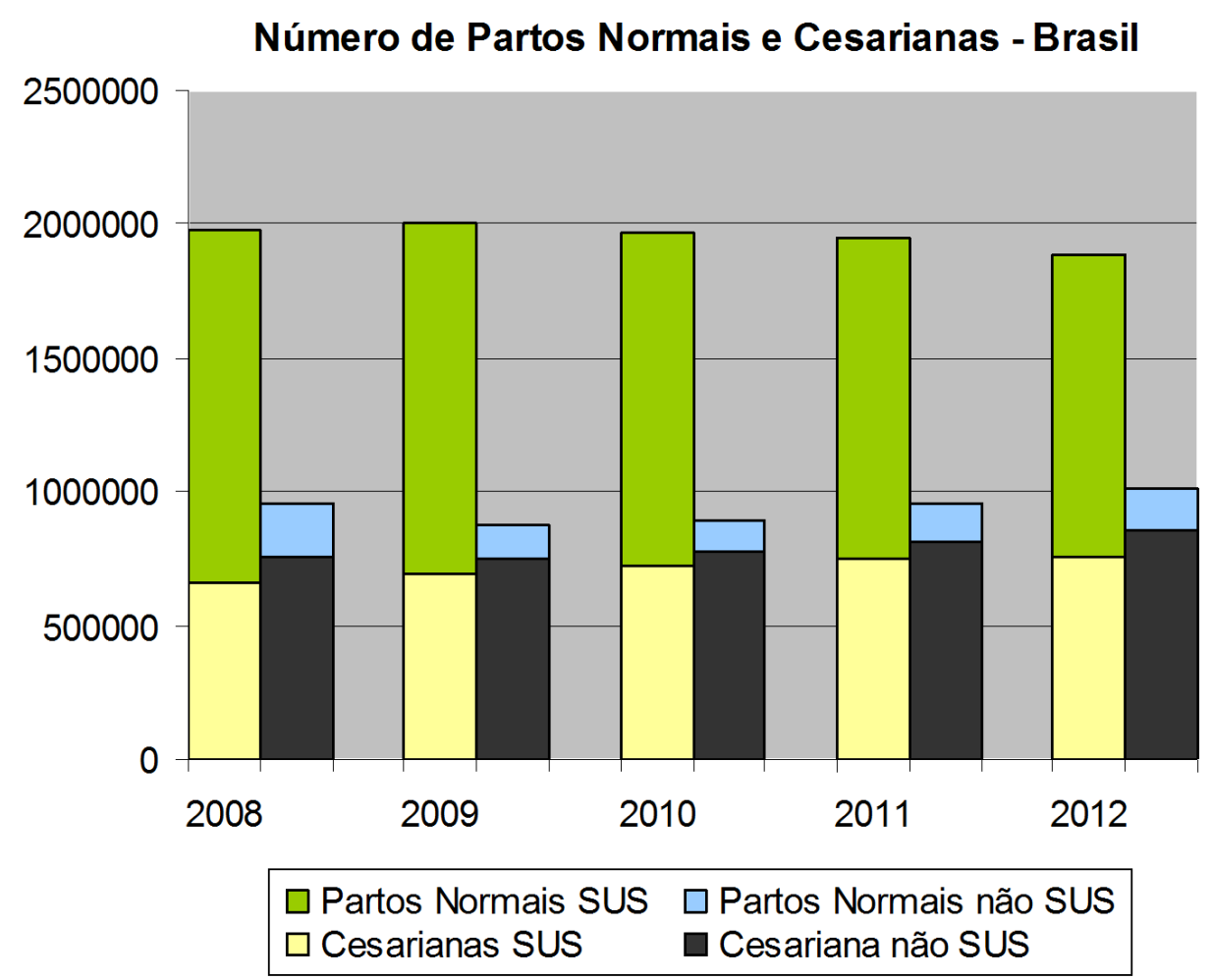

Gráfico 1. Número de partos normais e cesarianas, Brasil, 2008-2012.

A organização da assistência influencia as taxas de cesariana, que tendem a ser menores nos países com uma assistência obstétrica menos medicalizada, com maior atuação de midwives $^{52}$ - ofício equivalente ao desempenhado pelas obstetrizes formadas em curso de graduação no Brasil, sendo que atualmente há apenas um curso pioneiro desse tipo na Universidade de São Paulo - e/ou enfermeiras obstetras e com menor freqüência de intervençóes, como na Holanda, Nova Zelândia e países Escandinavos. ${ }^{53}$

Segundo a OMS, a assistência ao parto realizada por profissionais capacitados é o fator mais importante para diminuição da mortalidade materna. ${ }^{54}$ São atendentes qualificados para a assistência ao parto: médicos (inclusive MFCs), obstetrizes e enfermeiras obstetras. ${ }^{54}$ No Brasil, médicos e suas associaçóes demonstram grande preocupação com o parto de risco habitual atendido por obstetrizes e/ou enfermeiras obstetras, geralmente alegando que partos assistidos por esses profissionais teriam maior risco para a mãe e o concepto. Isso não é verificado na literatura, assim como é refutado por ela. ${ }^{55,56,57}$

Fatores ligados aos médicos especialistas assistentes no Brasil agem como forças geradoras do excesso de cesarianas: conveniência, agendamento, rapidez no parto, receio de processos por má prática e a crença de que as mulheres preferem e solicitam a cesariana. ${ }^{44,58}$ Também há adesão ideológica dos médicos à cesariana, como mostra o caso da epígrafe. Além disso, quando o médico faz o pré-natal, conveniência, facilidade de agendamento e rapidez no procedimento significam desdobramentos econômicos com melhor administração de outras atividades remuneradas e de tempo livre. ${ }^{59}$ Adicionalmente, com o persistente excesso de cesarianas por décadas no Brasil, provavelmente ocorreu (e continua ocorrendo) uma modificação no treinamento dos médicos obstetras no país: eles podem não estar desenvolvendo e exercitando habilidades clínicas para acompanhar um parto normal sem intervenções cirúrgicas. Ao não saberem mais manejar clinicamente partos, qualquer complicação e/ ou distócia, ou mesmo variação da normalidade tende a ser resolvida pela cirurgia, que em muitos casos poderia ser evitada com benefícios para a mulher e seu bebê. ${ }^{59}$ Todos esses fatores ajudam na compreensão do enorme número de violaçóes de direitos e de agressóes de várias ordens, bem como do grande volume de cesarianas desnecessárias que ocorrem atualmente no Brasil, tanto no setor privado como no sistema público de saúde. 


\section{Prevenção quaternária da violência obstétrica (V0): 0 que fazer e como}

Considerando-se a magnitude e a complexidade da VO no Brasil, são indicados dois tipos de açôes de prevenção quaternária: açóes individuais, familiares e comunitárias realizadas na APS, associadas ao pré-natal; e ações em maior escala (social, política e institucional).

Apesar da elevada cobertura de pré-natal $(98,7 \%)$ no Brasil, apenas $40 \%$ das mulheres afirmam ter recebido orientaçóes sobre práticas benéficas para o trabalho de parto, sendo maiores as orientaçôes sobre gravidade e sinais de risco. ${ }^{25,60}$ É importante mudar essa visão e instrumentalizar o MFC para que oriente as gestantes e famílias quanto aos benefícios do parto natural e às possíveis complicaçôes de intervençóes questionáveis ${ }^{60}$ a fim de contribuir para o empoderamento das mulheres e suas famílias e para que elas possam exigir um atendimento obstétrico seguro e digno. Dessa forma, enfrentar a VO exige que os profissionais de saúde assumam uma responsabilidade para além das rotinas de pré-natal e do entendimento biomédico da gestação e parturiçãao.

Um recurso subutilizado no Brasil é a construção de planos de parto, prática iniciada na década de 1970 entre mulheres europeias que reivindicavam controle sobre seu próprio corpo no contexto da crescente medicalização do parto ${ }^{61}$ Recomendado pela OMS ${ }^{62}$ sua elaboração convida a mulher, auxiliada por seus cuidadores, a refletir sobre práticas benéficas e danosas e sobre seus direitos durante o processo de parturiçáo.

O plano de parto exige um esforço da mulher para entender e expressar seus valores pessoais, medos e necessidades no processo parturitivo e facilita a comunicação dessas preferências aos profissionais da assistência. ${ }^{63,64}$ São vantagens descritas do uso do plano de parto a melhoria da comunicação com a equipe de assistência, a sensação de ter escolhas e maior controle durante o parto, fazendo com que a mulher esteja mais consciente das opçôes que tem durante o parto. ${ }^{52,64}$

Para a APS, o plano de parto traz a necessidade de se pensar o pré-natal como espaço importante de compartilhamento de informaçôes e pactuação de estratégias que mitiguem a situação de desamparo em que se transformou a assistência ao parto. Ajudar as usuárias a construírem seus planos promove a reflexão das equipes sobre a realidade da assistência ao parto na sua regiấo e sua atuação nesse cenário. Um forte vínculo entre equipe e gestante na APS pode minimizar os danos da descontinuidade de cuidado entre o pré-natal e parto, criando uma ponte entre a equipe cuidadora na APS e o local de assistência ao parto.

O plano de parto pode ser construído tanto de forma individual como coletivamente. No modo individual a mulher pode ser orientada a buscar informaçóes disponíveis e/ou o profissional (médico ou enfermeira) pode utilizar uma consulta para sua construção. No entanto, o modo coletivo, por meio de encontros de gestantes, muito comum na APS brasileira, talvez seja mais produtivo. Nesses espaços coletivos é interessante partir do universo referencial das mulheres, suas histórias e percepçôes, ao invés de construir uma fala linear e/ou unidirecional. Partindo de uma primeira rodada de relatos, amplia-se o debate. É importante estar aberto para a escuta e uma aliança com as potencialidades das mulheres. A discussão de todas as rotinas, intervençôes e práticas pode requerer mais de um encontro do grupo de gestantes. A dificuldade que muitas mulheres enfrentam para se ausentar do domicílio, devido ao cuidado com filhos menores, pode ser mitigada designando um profissional como cuidador, facilitando o acesso às multíparas. No Quadro 4 apresenta-se um roteiro para elaboração de um plano de parto.

Pode-se discutir que as mulheres atualmente sejam mais permissíveis a condutas médicas desnecessárias e que algumas até as desejem, por terem introjetado culturalmente um modelo de assistência que é oferecido de forma hegemônica. ${ }^{64,65}$ Cabe lembrar que, mesmo com o modelo vigente, a maioria das brasileiras não prefere a cesariana para o nascimento de seus filhos ${ }^{66}$ e supóe-se que ninguém deseje ou anseie por procedimentos dolorosos e ineficazes. Uma vez informadas sobre possíveis alternativas e direitos, supõe-se que as mulheres não somente optariam, mas também lutariam por uma assistência obstétrica com garantias de integridade física e emocional para si e para seus bebês.

É importante que se esclareça que refletir sobre e escrever um plano de parto não garante o cumprimento, pelo serviço de atenção ao parto, daqueles desejos. $\mathrm{Na}$ atual conjuntura é provável que a reação dos profissionais não seja receptiva. Uma das possíveis desvantagens do plano de parto é, justamente, o aumento da tensão e de conflitos entre provedores e mulheres ${ }^{64}$ e a criação de uma atmosfera de desconfiança entre os envolvidos. ${ }^{63}$ Outra desvantagem citada é que aumenta a insatisfação das mulheres ao não conseguirem ter a assistência ou o parto que planejaram. ${ }^{63,64}$ Porém, quanto mais as mulheres requisitarem atendimento digno mais difícil será que se perpetue a atual situação. Destaca-se que a elaboração de planos de parto deve ser estimulada por equipes que acreditem nas evidências e nos direitos das mulheres, ou corre-se o risco de se transformar tal proposta em mais um papel a ser preenchido de forma acrítica, burocrática e sem valor. ${ }^{64}$ 
Quadro 4. Questões para a construção/discussão de um plano de parto.

\section{INÍCIO DO TRABALHO DE PARTO \\ PLANO DE PARTO \\ 1) Importância de só ir para a maternidade em trabalho de parto ativo já que internações precoces podem desencadear uma cascata de intervenções. \\ 2) Direito a acompanhante (Lei 11.108/2005) em todas as dependências da maternidade: sala de parto, centro cirúrgico e alojamento conjunto.}

\section{DURANTE 0 TRABALHO DE PARTO}

1) Escolha do Local de Parto - Domicílio, Centro de Parto Normal, Casa de Parto, Maternidade (entender as diferenças entre estas instituições e cobrar das mesmas a divulgação de seus indicadores).

2) Procedimentos questionáveis para os quais não há evidência de benefício:

- Tricotomia (raspagem pelos pubianos), enema, acesso venoso e ocitocina rotineiros, jejum, amniotomia

3) Auxiliam a tolerar as dores do trabalho de parto e tem se mostrado benéficos: liberdade de movimento, meios não farmacológicos para alívio da dor (banho quente massagem).

4) Se sentir necessidade de analgesia de parto solicitar consentimento informado antes de realizá-la.

\section{DURANTE O PARTO}

(Conhecer indicações de cesariana e funcionamento do partograma).

1) Liberdade para escolher posição de parto e priorizar posição verticalizada ou lateralizada (cócoras, semi-sentada, em quarto apoios).

2) Não aceitar pernas presas em perneiras ou estribos ou posição de litotomia completa.

3) Orientar preparo perineal a partir de 34 semanas com óleo vegetal e evitar puxos induzidos. A mulher empurra quando tem vontade. Isso diminui as chances de laceração perineal.

4) Episiotomia não deve ser realizada rotineiramente.

5) Clampeamento oportuno do cordão umbilical.

6) 0 pai ou acompanhante podem ser incluídos na cena do parto e solicitarem cortar o cordão.

7) Contato precoce pele-a-pele entre mãe e bebê.

8) Bebê amamentado assim que possível.

\section{PÓS-PARTO}

1) Alojamento conjunto 24 horas.

2) Livre demanda ao aleitamento materno. Questionar complementação com leite artificial.

Fonte: elaboração dos autores.

Dispóe-se atualmente de inúmeros recursos audiovisuais que podem auxiliar no estímulo a reflexóes e debates sobre planos de parto. O filme "Um dia de Vida" do Ministério da Saúde mostra a realidade de uma Casa de Parto em Ceres, Goiás, e a trajetória das famílias ali atendidas. O documentário "O SUS que dá certo: Parto e Nascimento humanizado no Hospital Sofia Feldman" retrata o processo de trabalho e o envolvimento de profissionais e de usuárias. Outro documentário, "O Renascimento do parto", denuncia a negação às mulheres do direito à informação e autonomia e divulga outra narrativa possível para o parto: uma experiência segura, prazerosa e única.

Além dos filmes citados, os profissionais podem acessar a biblioteca Cochrane, com revisões sistemáticas sobre a maioria das condutas obstétricas de rotina. Há sítios que fazem uma "tradução" das evidências científicas para profissionais e usuárias, como, por exemplo, o blog da obstetra Melania Amorim e o sítio Childbirth Connection. Há blogs de grupos de apoio à gestação e ao parto com relatos de parto, como a iniciativa Parto no Rio e a proposta 'BH pelo parto normal'. Portanto, a discussão e a elaboração de planos de parto com as gestantes é uma estratégia importante e de fácil operacionalização de açóes de prevenção quaternária.

A atuação dos profissionais da APS e suas associaçôes se justifica também fora do ambiente dos serviços de APS. Embora a prevenção quaternária tenha nascido no ambiente do cuidado clínico na APS, deve ir além da clínica, construindo formas de organização social do cuidado profissional que signifiquem diminuiçáo de danos/hipermedicalizaçáo. Por exemplo, a construção de sistemas universais públicos de saúde com APS forte como função filtro pode ser considerada uma ação organizativa/política de prevenção quaternária. ${ }^{67,68}$ Nesse mesmo sentido, a prevenção quaternária da VO no Brasil pode se dar através da urgente reforma das práticas obstétricas, com a inserção de outros profissionais habilitados no cuidado ao parto de risco habitual e utilizando boas práticas obstétricas, seja nas instituições em funcionamento, seja em outros locais de cuidado ao parto/nascimento. ${ }^{52} \mathrm{~A}$ atuaçáo dos médicos obstetras especialistas seria importante e otimizada em casos de risco não habitual e como segunda linha de cuidado, acionada quando necessário. ${ }^{59}$ 
A situação exige a união de esforços para a melhoria da assistência obstétrica. Na prática, é o caso de as associaçôes (como a Sociedade Brasileira de Medicina de Família e Comunidade) se pronunciarem com seu apoio e ativismo social e político à causa do combate à $\mathrm{VO}$, à redução do excesso de cesarianas e à promoção de ambientes, serviços e atividades profissionais de boas práticas obstétricas. O atendimento a partos de risco habitual por MFCs é uma realidade em diversos países, inclusive com menores taxas de episiotomias. ${ }^{69}$

As demandas de mulheres organizadas têm ecoado aos poucos nas políticas federais. A Rede Cegonha ${ }^{70}$ pretende implantar 280 Centros de Parto Normal até 2015, promovendo uma adequação da ambiência para o trabalho de parto e parto e priorizando a atuaçáo de enfermeiras obstetras e obstetrizes no atendimento de partos de risco habitual, medida reconhecida mundialmente por seus benefícios em reduzir intervençôes desnecessárias e número de cesarianas. ${ }^{52}$ Tais medidas, no entanto, encontram muita resistência, principalmente por parte de profissionais médicos e suas entidades, devido ao deslocamento de poder que promovem e pela revisão de práticas que implicam. Torna-se necessário, portanto, que os MFCs e outros profissionais da APS e suas associaçôes se articulem aos movimentos de mulheres e ocupem os espaços de participação social no SUS, tanto as Conferências Municipais de Saúde e os espaços da Rede Cegonha, como os Fóruns Perinatais, ${ }^{70}$ para cobrar a elaboração e o monitoramento dos indicadores propostos pelos Planos de Açáo dos Grupos Condutores da Rede Cegonha e outros considerados importantes, como os incluídos no "termômetro de segurança materna".

Tal política tem grande importância se considerarmos como pode ser indutora de novas subjetividades dentro do SUS. Sabe-se que, com a diminuição da pobreza e a inclusão no mercado de segmentos sociais ansiosos pelo consumo de bens e serviços, há migração de mulheres para o setor suplementar em busca de cesarianas. ${ }^{71}$ É também realidade que mulheres de classe média, devido à falta de opção para parto normal no setor privado, buscam serviços públicos, com atendimento pautado em evidências, direitos e respeito. ${ }^{72}$

Conhecer e apoiar os exemplos pioneiros de instituiçóes e serviços nos quais a VO está minimizada ou ausente, como o Hospital Sofia Feldman e casas de parto, bem como incentivar o surgimento de novas alternativas assistenciais às gestantes e a reforma das práticas das maternidades em funcionamento no Brasil, são tarefas urgentes e difíceis.

\section{Conclusão}

A prevenção quaternária frente à violência obstétrica no Brasil requer a participação dos profissionais e suas associações em duas frentes: a primeira envolve a atuação clínica no cuidado e apoio às gestantes e puérperas, bem como a elaboração participativa de planos de parto. A segunda requer suporte e participação social para que sejam atendidas as reivindicaçôes de humanizaçáo da assistência ao pré-natal e parto, provenientes dos movimentos de mulheres, de modo a impactar significativamente a violência obstétrica no sistema de saúde do Brasil.

\section{Referências}

1. Entrevista com Adelir Carmen Lemos de Góes [Internet]. Cientista que virou mãe; 2012 [acesso em 2015 Jun 17]. Disponível em: http://www.cientistaqueviroumae.com.br/2014/04/entrevista-com-adelir-carmen-lemos-de.html

2. Justiça do RS manda grávida fazer cesariana contra sua vontade [Internet]. São Paulo: Folha de São Paulo; 2014 [acesso em 2015 Jun 17]. Disponível em: http://www1.folha.uol.com.br/cotidiano/2014/04/1434570-justica-do-rs-manda-gravida-fazer-cesariana-contra-sua-vontade.shtml

3. Rattner D. Humanização na atenção a nascimentos e partos: ponderações sobre políticas públicas. Interface (Botucatu). 2009;13(1 supl):759-768. http://dx.doi.org/10.1590/S1414-32832009000500027.

4. Venturi G, Godinho T. Mulheres brasileiras e gênero nos espaços público e privado: uma década de mudanças na opinião pública. São Paulo: Editora Fundação Perseu Abramo/SESC-SP; 2013.

5. Bentzen N, editor. Wonca dictionary of general/family practice. Copenhagen: Maanedsskrift for Praktisk Laegegerning; 2003.

6. Ministério da Saúde do Brasil (BR). DATASUS: Sistema de Informações de Nascidos Vivos [Internet]. Ano [acesso em 2014 July 1]. Disponível em: http://www2.datasus.gov.br/DATASUS/index.php?area=0205\&VObj=http://tabnet.datasus.gov.br/cgi/deftohtm.exe?sinasc/cnv/nv

7. Ley Orgánica sobre el Derecho de las Mujeres a una Vida Libre de Violencia [Internet]. Gaceta Oficial 38.647. [Acesso em 2015 Jun 17 ]. Disponível em: http://venezuela.unfpa.org/doumentos/Ley_mujer.pdf.

8. Bowser D, Hill K. Exploring evidence for disrespect and abuse in facility-based childbirth: report of a landscape analysis. Bethesda, Maryland: USAID-TRAction Project; 2010. 
9. D'Oliveira AF, Diniz SG, Schraiber LB. Violence against women in health-care institutions: an emerging problem. Lancet. 2002;359(9318):16811685. http://dx.doi.org/10.1016/S0140-6736(02)08592-6. PMid:12020546.

10. Goer H. Cruelty in maternity wards: fifty years later. J Perinat Educ. 2010;19(3):33-42. http://dx.doi.org/10.1624/105812410X514413. PMid:21629381.

11. Comité de América Latina y el Caribe para la Defensa de los Derechos de la Mujer (CLADEM); Centro Legal para Derechos Reproductivos y Políticas Públicas (CRLP). Silencio y cumplicidad: violencia contra la mujer em los servicios de salud em el Peru Lima. Lima: CLADEM; 1998.

12. National Health Service (NHS). NHS Safety Thermometer [internet]. 2013 [acesso em 2015 Jun 17]. Disponível em: http://www.safetythermometer.nhs.uk/index.php?option=com_content\&view=article\&id=11:maternity-homepage\&catid=2:uncategorised\&ltemid=285

13. Souza JP, Pileggi-Castro C. Sobre o parto e o nascer: a importância da prevenção quaternária. Cad Saude Publica. 2014;30(Supl):S11-S13. http://dx.doi.org/10.1590/0102-311XPE02S114.

14. Organização Mundial da Saúde (OMS). Assistência ao parto normal: um guia prático: relatório de um grupo técnico. Genebra: OMS; 1996.

15. Ministério da Saúde (BR). Parto, aborto e puerpério: assistência humanizada à mulher [Internet]. Brasília: Ministério da Saúde; 2001 [acesso em 2015 Jun 17]. 93 p. Disponível em: http://bvsms.saude.gov.br/bvs/publicacoes/cd04_13.pdf

16. Dawood F, Dowswell T, Quenby S. Intravenous fluids for reducing the duration of labour in low risk nulliparous women. Cochrane Database Syst Rev. 2013;6(6):CD007715. http://dx.doi.org/10.1002/14651858. PMid:23780639.

17. Costley PL, East CE. Oxytocin augmentation of labour in women with epidural analgesia for reducing operative deliveries. Cochrane Database Syst Rev. 2012;5(5):CD009241. http://dx.doi.org/10.1002/14651858. PMid:22592738.

18. Fraser WD, Turcot L, Krauss I, Brisson-Carrol G. Amniotomy for shortening spontaneous labour. Cochrane Database Syst Rev. 2013;(6). http://dx.doi.org/10.1002/14651858.CD006167.pub4.

19. Lawrence A, Lewis L, Hofmeyr GJ, Dowswell T, Styles C. Maternal positions and mobility during first stage labour. Cochrane Database Syst Rev. 2009;(2):CD003934. PMid:19370591.

20. Priddis $\mathrm{H}$, Dahlen $\mathrm{H}$, Schmied V. What are the facilitators, inhibitors, and implications of birth positioning? A review of the literature. Women Birth. 2012;25(3):100-106. http://dx.doi.org/10.1016/j.wombi.2011.05.001. PMid:21664208.

21. Räisänen S, Vehviläinen-Julkunen K, Gisler M, Heinonen S. A population-based register study to determine indications for episiotomy in Finland. Int J Gynaecol Obstet. 2011;115(1):26-30. http://dx.doi.org/10.1016/j.ijgo.2011.05.008. PMid:21767841.

22. Carroli G, Mignini L. Episiotomy for vaginal birth. Cochrane Database Syst Rev. 2009;(1):CD000081. PMid:19160176.

23. Moiety FMS, Azzam AZ. Fundal pressure during the second stage of labor in a tertiary obstetric center: a prospective analysis. J Obstet Gynaecol Res. 2014;40(4):946-953. http://dx.doi.org/10.1111/jog.12284. PMid:24428496.

24. Verheijen EC, Raven JH, Hofmeyr GJ. Fundal pressure during the second stage of labour. Cochrane Database Syst Rev. 2009;7(4):CD006067. PMid:19821352.

25. Singata M, Tranmer J, Gyte GM. Restricting oral fluid and food intake during labour. Cochrane Database Syst Rev. 2010;20(1):CD003930. PMid:20091553.

26. Lawrence A, Lewis L, Hofmeyr GJ, Styles C. Maternal positions and mobility during first stage labour. Cochrane Database Syst Rev. 2013;(10):1-158. http://dx.doi.org/10.1002/14651858.CD003934.pub4.

27. Diniz CSG, d'Orsi E, Domingues RMSM, Torres JA, Dias MAB, Schneck CA, et al. Implementação da presença de acompanhantes durante a internação para o parto: dados da pesquisa nacional Nascer no Brasil. Cad Saude Publica. 2014;30(Supl):S140-S153. http://dx.doi.org/10.1590/0102-311X00127013.

28. Viellas EF, Domingues RMSM, Dias MAB, Gama SGN, Theme MM Fa, Costa JV, et al. Assistência ao pré-natal no Brasil. Cad Saude Publica. 2014;30(Supl):S85-S100.

29. Villar J, Carroli G, Zavaleta N, Donner A, Wojdyla D, Faundes A, et al. Maternal and neonatal individual risks and benefits associated with caesarean delivery: multicentre prospective study. BMJ. 2007;335(7628):1025. http://dx.doi.org/10.1136/bmj.39363.706956.55. PMid:17977819.

30. Belizán JM, Althabe F, Barros FC, Alexander S, Showalter E, Griffin A, et al. Rates and implications of caesarean sections in Latin America: ecological study. BMJ. 1999;319(7222):1397-1400. http://dx.doi.org/10.1136/bmj.319.7222.1397. PMid:10574855.

31. Ye J, Betrán AP, Guerrero Vela M, Souza JP, Zhang J. Searching for the optimal rate of medically necessary cesarean delivery. Birth. 2014;41(3):237-244. http://dx.doi.org/10.1111/birt.12104 PMid:24720614.

32. Villar J, Valladares E, Wojdyla D, Zavaleta N, Carroli G, Velazco A, et al. Caesarean delivery rates and pregnancy outcomes: the 2005 WHO global survey on maternal and perinatal health in Latin America. Lancet. 2006;367(9525):1819-1829. http://dx.doi.org/10.1016/S0140-6736(06)68704-7. PMid:16753484.

33. Villar J, Carroli G, Zavaleta N, Donner A, Wojdyla D, Faundes A, et al. Maternal and neonatal individual risks and benefits associated with caesarean delivery: multicentre prospective study. BMJ. 2007;335(7628):1025. http://dx.doi.org/10.1136/bmj.39363.706956.55. PMid:17977819.

34. Morlando M, Sarno L, Napolitano R, Capone A, Tessitore G, Maruotti GM, et al. Placenta accreta: incidence and risk factors in an area with a particularly high rate of cesarean section. Acta Obstet Gynecol Scand. 2013;92(4):457-460. http://dx.doi.org/10.1111/aogs.12080. PMid:23347183.

35. Hansen AK, Wisborg K, Uldbjerg N, Henriksen TB. Risk of respiratory morbidity in term infants delivered by elective caesarean section: cohort study. BMJ. 2008;336(7635):85-87. http://dx.doi.org/10.1136/bmj.39405.539282.BE. PMid:18077440. 
36. Tita AT, Landon MB, Spong CY, Lai Y, Leveno KJ, Varner MW, et al. Timing of elective repeat cesarean delivery at term and neonatal outcomes. N Engl J Med. 2009;360(2):111-120. http://dx.doi.org/10.1056/NEJMoa0803267. PMid:19129525.

37. Gyamfi-Bannerman C, Fuchs KM, Young OM, Hoffman MK. Nonspontaneous late preterm birth: etiology and outcomes. Am J Obstet Gynecol. 2011;205(5):456.e1-456.e6. http://dx.doi.org/10.1016/j.ajog.2011.08.007. PMid:22035950.

38. Bettiol H, Rona RJ, Chinn S, Goldani M, Barbieri MA. Factors associated with preterm births in southeast Brazil: a comparison of two birth cohorts born 15 years apart. Paediatr Perinat Epidemiol. 2000;14(1):30-38. http://dx.doi.org/10.1046/j.1365-3016.2000.00222.x. PMid:10703032.

39. Leal M, Silva AAM, Dias MAB, Gama SGN, Rattner D, Moreira ME, et al. Birth in Brazil: national survey into labour and birth. Reprod Health. 2012;15(9):1-8. http://dx.doi.org/10.1186/1742-4755-9-15. PMid: 22913663.

40. Hyde MJ, Modi N. The long-term effects of birth by caesarean section: the case for a randomised controlled trial. Early Hum Dev. 2012;88(12):943949. http://dx.doi.org/10.1016/j.earlhumdev.2012.09.006. PMid:23036493.

41. Darmasseelane K, Hyde MJ, Santhakumaran S, Gale C, Modi N. Mode of delivery and offspring body mass index, overweight and obesity in adult life: a systematic review and meta-analysis. PLoS One. 2014;9(2):e878-e896. http://dx.doi.org/10.1371/journal.pone.0087896. PMid:24586295.

42. Bager P, Wohlfahrt J, Westergaard T. Caesarean delivery and risk of atopy and allergic disease: meta-analyses. Clin Exp Allergy. 2008;38(4):634-642. http://dx.doi.org/10.1111/j.1365-2222.2008.02939.x. PMid:18266879.

43. Cho CE, Norman M. Cesarean section and development of the immune system in the offspring. Am J Obstet Gynecol. 2013;208(4):249-254. http://dx.doi.org/10.1016/j.ajog.2012.08.009. PMid:22939691.

44. Barros AJ, Santos IS, Matijasevich A, Domingues MR, Silveira M, Barros FC, et al. Patterns of deliveries in a Brazilian birth cohort: almost universal cesarean sections for the better-off. Rev Saude Publica. 2011;45(4):635-643. http://dx.doi.org/10.1590/S0034-89102011005000039. PMid:21670862.

45. Rattner D. Sobre a hipótese de estabilização das taxas de cesárea do Estado de São Paulo, Brasil. Rev Saude Publica. 1996;30(1):19-33. http://dx.doi.org/10.1590/S0034-89101996000100004. PMid:9008919.

46. Faúndes A, Pádua KS, Osis MJD, Cecatti JG, Sousa MH. Opinião de mulheres e médicos brasileiros sobre a preferência pela via de parto. Rev Saude Publica. 2004;38(4):488-494. http://dx.doi.org/10.1590/S0034-89102004000400002. PMid:15311287.

47. Mandarino NR, Chein MB, Monteiro F Jr, Brito LM, Lamy ZC, Nina VJ, et al. Aspectos relacionados à escolha do tipo de parto: um estudo comparativo entre uma maternidade pública e outra privada, em São Luís, Maranhão, Brasil. Cad Saude Publica. 2009;25(7):1587-1596. http://dx.doi.org/10.1590/S0102-311X2009000700017. PMid:19578580.

48. Pang MW, Leung TN, Lau TK, Hang Chung TK. Impact of first childbirth on changes in women's preference for mode of delivery: follow-up of a Iongitudinal observational study. Birth. 2008;35(2):121-128. http://dx.doi.org/10.1111/j.1523-536X.2008.00225.x. PMid:18507583.

49. Faisal-Cury A, Menezes PR. Fatores associados à preferência por cesariana. Rev Saude Publica. 2006;4(2):21-26.

50. Cardoso JE, Barbosa RHS. O desencontro entre desejo e realidade: a "indústria" da cesariana entre mulheres de camadas médias no Rio de Janeiro, Brasil. Physis. 2012;22(1):35-52. http://dx.doi.org/10.1590/S0103-73312012000100003.

51. O'Dougherty M. Plot and irony in childbirth narratives of middle-class Brazilian women. Med Anthropol Q. 2013;27(1):43-62. http://dx.doi.org/10.1111/maq.12015. PMid:23674322.

52. Norman AH, Tesser CD. Obstetrizes e enfermeiras obstetras no Sistema Único de Saúde e na Atenção Primária à Saúde: por uma incorporação sistêmica e progressiva. Rev Bras Med Fam Comunidade. 2015;10(34):1-7. http://dx.doi.org/10.5712/rbmfc10(34)1106.

53. Patah LE, Malik AM. Modelos de assistência ao parto e taxa de cesária em diferentes países. Rev Saude Publica. 2011; 45(1):185-194. http://dx.doi.org/10.1590/S0034-89102011000100021. PMid:21181056.

54. Reducción de la mortalidad materna: declaración conjunta OMS/FNUAP/UNICEF/ Banco Mundial [Internet]. Ginebra: Organización Mundial de la Salud; 1999 [acesso em 2015 Jun 17]. http://whqlibdoc.who.int/publications/1999/9243561952_spa.pdf?ua=1

55. Mouta RJO, Progianti JM. Estratégias de luta das enfermeiras da Maternidade Leila Diniz para implantação de um modelo humanizado de assistência ao parto. Texto \& Contexto - Enfermagem. 2009;18(4):731-740. http://dx.doi.org/10.1590/S0104-07072009000400015.

56. Narchi NZ. Análise do exercício de competências dos não médicos para atenção à maternidade. Saúde e Sociedade. 2010;19(1):147-158. http://dx.doi.org/10.1590/S0104-12902010000100012

57. Sandall J, Soltani H, Gates S, Shennan A, Devane D. Midwife-led continuity models versus other models of care for childbearing women. Cochrane Database Syst Rev. 2013;8:1-107. http://dx.doi.org/10.1002/14651858.CD004667. pub3. PMid:23963739.

58. Faúndes A, Cecatti JG. A operação cesárea no Brasil: incidência, tendências, causas, conseqüências e propostas de ação. Cad Saude Publica. 1991;7(2):150-173. http://dx.doi.org/10.1590/S0102-311X1991000200003. PMid:15830039.

59. Tesser CD, Knobel R, Rigon T, Bavaresco GZ. Os médicos e o excesso de cesárias no Brasil. Sau \& Transf Soc. 2011:2(1):4-12. [acesso em 2015 Jun 17]. Disponível em: http://periodicos.incubadora.ufsc.br/index.php/saudeetransformacao/article/view/1088

60. Klein MC, Kaczorowski J, Tomkinson J, Hearps S, Baradaran N, Brant R. Family physicians who provide intrapartum care and those who do not: very different ways of viewing childbirth. Can Fam Physician. 2011;57(4):e139-e147. PMid:21490345.

61. Penny Simkin P. Birth plans: after 25 years, women still want to be heard. Birth. 2007;34(1):49-51. http://dx.doi.org/10.1111/j.1523-536X.2006.00126.x. PMid:17324178. 
62. World Health Organization (WHO). Birth and emergency preparedness in antenatal care. Intergrated management of pregnancy and childbirth (IMPAC). Geneva: Department of Making Pregnancy Safer, WHO; 2006 [acesso em 2015 jun 17].

Disponível em: http://www.who.int/reproductivehealth/publications/maternal_perinatal_health/emergency_preparedness_antenatal_care.pdf

63. Bailey JM, Crane P, Nugent CE. Childbirth education and birth plans. Obstet Gynecol Clin North Am. 2008;35(3):497-509. http://dx.doi.org/10.1016/j.ogc.2008.04.005. PMid:18760232.

64. Lothian J. Birth plans: the good, the bad, and the future. J Obstet Gynecol Neonatal Nurs. 2006;35(2):295-303. http://dx.doi.org/10.1111/j.1552-6909.2006.00042.x. PMid:16620258.

65. Declercq E, Sakala C, Corry M, Applebaum S, Risher P. Listening to mothers: Report of the First National US Survey of Women's Childbering Experiences. New York: Maternity Center Association; 2002.

66. Domingues RMSM, Dias MAB, Nakamura-Pereira M, Torres JA, d'Orsi E, Pereira APE, et al. Processo de decisão pelo tipo de parto no Brasil: da preferência inicial das mulheres à via de parto final. Cad Saude Publica. 2014;30(Supl):S101-S116. http://dx.doi.org/10.1590/0102-311X00105113.

67. Hart JT. The inverse care law. Lancet. 1971;1(7696):405-412. http://dx.doi.org/10.1016/S0140-6736(71)92410-X. PMid:4100731.

68. Gérvas J, Pérez Fernández M. El fundamento científico de la función de filtro del médico general. Rev Bras Epidemiol. 2005;8(2):205-218. http://dx.doi.org/10.1590/S1415-790X2005000200013.

69. Abenhaim HA, Welt M, Sabbah R, Audibert F. Obstetrician or family physician: are vaginal deliveries managed differently? J Obstet Gynaecol Can. 2010;29(10):801-805

70. Ministério da Saúde (BR). Portaria GM/MS no 1459, de 24 de junho de 2011. Institui no âmbito do SUS, a Rede Cegonha. Diário Oficial da União. Brasília, 2011 [acesso em 2015 jun 17]. Disponível em: http://bvsms.saude.gov.br/bvs/saudelegis/gm/2011/prt1459_24_06_2011.html

71. Aquino EML. Para reinventar o parto e o nascimento no Brasil: de volta ao futuro. Cad Saude Publica. 2014;30(Supl):S8-S10. http://dx.doi. org/10.1590/0102-311XPE01S114.

72. Brasil L. Maternidade Pública Sofia Feldman atrai clientes de planos de saúde [Internet]. Belo Horizonte: Revista VejaBH; 2013 [acesso em 2013 Feb 2]. Disponível em: http://vejabh.abril.com.br/edicoes/maternidade-publica-sofia-feldman-atrai-clientes-planos-saude-733707.shtml 